\title{
Anti-Bacterial Effect of Venom Extracted from Pardosa oakleyi (Family Lycosidae) and Silk Retrieved from Crossopriza lyoni (Family Pholcidae)
}

\author{
Hafiz Muhammad Tahir ${ }^{1}$, Breeha Kazmi ${ }^{2}$ and Iram Liaqat ${ }^{3 *}$ \\ ${ }^{1}$ Department of Zoology, GC University Lahore, Pakistan \\ ${ }^{2}$ Department of Zoology, University of Sargodha, Sargodha, Pakistan \\ ${ }^{3}$ Department of Zoology, GC University Lahore \\ *Corresponding Author: Iram Liaqat, Assistant Professor, Department of Zoology, GC University, Lahore, Pakistan.
}

Received: June 05, 2019; Published: September 16, 2019

DOI: $10.31080 /$ ASMI.2019.02.0373

\begin{abstract}
Spiders are widely known and abundantly present successful predators because they are surprisingly armed with potent cocktail of venom along with the multifunctional tangled webs. In current research, venom was recovered from Pardosa oakleyi (Family Lycosidae) and silk was recovered from Crossopriza lyoni (Family Pholcidae). Study was aimed to partially characterize the venom of $P$. oakleyiand for estimation of antibacterial potency of venom and silk. Four pathogenic strains of bacteria were used i.e., Gram positive (Staphylococcus sp. and Streptococcus sp.) and Gram negative (Acinetobacter sp. and Pasteurella sp.). Results revealed that the venom of P. oakleyi comprised of relatively high molecular weight peptides ranging from $155 \mathrm{kDa}$ to $43 \mathrm{kDa}$. And susceptibility tests indicated that the crude venom was ineffective against all tested strains. Althoughthe silk of $C$. lyoni exhibited significant bacteriostatic action against Acinetobacter sp. and Streptococcus sp.

Keywords: Characterization; Bacteriostatic Action; Peptides; Susceptibility Test; Crude Venom; Gram Positive and Gram Negative Strains
\end{abstract}

\section{Abbreviation}

SDS PAGE: Sodium Dodecyl Sulfate Polyacrylamide GelElectrophoresis.

\section{Introduction}

The rapid development of resistance in bacteria is taking place throughout the world which is threatening the effectiveness of antibiotics, the savior of millions of lives $[1,2]$. Several decades after initially patients were cured using antibiotics, bacterial infections have again turned into a tenacious and alarming threat [3]. Excessive utilization, mishandling and unsystematic use of broad-spectrum antibiotics are the major contributors in the emergence of resistivity among bacterial strains $[4,5]$. Almost all clinically significant bacterial pathogens show resistivity against antibiotics. The decrease in effectiveness of antibiotics in treating common infec- tions and the advent of novel untreatable strains indicates that we are at the edge of a post-antibiotic era [6].

The regular and prompt development of new interventions with antibacterial activity is the need of time to deal all challenges related to antibiotic resistivity in bacteria. Natural products are surpassing the synthetic products and providing foundation for innovation of chemical diversities including natural antibiotics [7]. These natural antibiotics are reported to be more effective, safer and have less side-effects on human health and environment as compared to synthetic antibiotics [8].

In phylum Arthropoda, spiders are one of the most diverse group with approximately 100,000 existing species [9]. Spiders 
are amazingly armed with potent cocktail of venom along with the multifunctional tangled webs. Spider venom consist of a wide range of biologically active compounds including various peptides, proteins, enzymes, salts and small organic molecules [10,11]. All the toxins in spider venom are intended to block variety of receptors, channels, membranes and diverse enzymes of invertebrates and vertebrates [12].

In past minor work has been done on isolation and applications of peptides extracted from spider venom. Owing to this insufficiency in research, the number of discovered peptides is reasonably less [11]. Usually spider venom contains peptides up to $25 \%$ by weight with great diversity of almost 12 million types of discovered peptides $[13,14]$. Moreover, peptidic content of venom is highly selective or specific in targeting. This property of venom peptides can lead to development of novel therapeutics $[15,16]$.

Moreover, spider silk comprises off diverse compounds such as potassium nitrate, bisphosphonate peptides [17]. Presently, spider silk proof itself a marvelous gift of nature by exhibiting its multiple potentials. It is wonderful combination of strength and extensibility [18]. Spider silk can be amazingly useful in medical field because of its compatibility with living tissues of humans $[17,18]$ and most importantly spider silk contain number of antimicrobial compounds such as potassium nitrate, bisphosphonate peptides and phospholipids hydrates with obvious antibacterial potential $[19,20]$. Another important constituent of spider silk is potassium hydrogen phosphate. It basically releases protons in aqueous medium to make the silk acidic ( $\mathrm{pH} 4)$, which inhibit the microbial growth [21]. Furthermore, the lipid present in spider silk holds 12-methyltetradecanoic acid and 14-methylhexadecanoic acid that restrict microbial growth [21]. Silk contains active agents which do not allow bacterial strains to gain resistivity against natural antibacterial agent [22]. These properties make spider silk an admirable candidate for innovation in antimicrobial drug discovery.

Hence the venom and silk, extracted from spiders, could be one of the most effective ones among fewer natural resources to be discovered as antimicrobial drugs, as they are surprisingly remarkable weapons of spiders and so far very little or no attention has been paid to them in this context.
Therefore, this study was conducted in order to check antibacterial potential of potent peptides present in spider venom (Pardosa oakleyi) and spider silk (Crossopriza lyoni). For this purpose partial characterization of venom (Pardosa oakleyi) was also done and to evaluate antibacterial potential of venom and silk four different pathogenic strains (including gram positive and gram negative both) were used. Significant bacteriostatic property by these peptides may lead to invention of novel therapeutics.

\section{Materials and Methods}

- Spider collection and venom extraction: Spiders (Pardosa oakleyi) were collected from citrus orchard and agricultural fields. Spiders were collected in separate aerated vials to avoid cannibalism among them. Chelicerae along with venom glands were separated after immobilization of spiders through freezing [23]. After that crude sample was prepared by homogenizing extracted chelicerae in $0.5 \mathrm{ml}$ of tris $\mathrm{HCl}$ buffer of pH 8.2 and molarity 0.05 M [24] and was centrifuged (MPW-352R) at 15,000 rpm for 20 minutes at $4^{\circ} \mathrm{C}$. Then supernatant extracted through suction pipette was frozen at $-20^{\circ} \mathrm{C}$ in Ultra freezer [24].

- Characterization of venom by sodium dodecyl sulphate (SDS) gel electrophoresis: Characterization involves fractionation of a variety of constituent proteins on the basis of their molecular weights. SDS polyacrylamide gel electrophoresis was performed to separate different venom fractions by following protocol of Sambrook and Russle [25]. Gel was categorized as 5\% staking gels and $12 \%$ resolving gel on the basis of different percentages. Supernatant containing venom sample melted within 5 minutes and were mixed in equal ratio (1:1) with $2 X$ SDS loading buffer (100M tris-Cl, $\mathrm{pH}$ 6.8) before heating at $100^{\circ} \mathrm{C}$.

- $\quad$ After running the sample with ladder (EZ ${ }^{\mathrm{TM}}$-Prestained protein Ladder Marker), gel was stained in Comassi brilliant blue staining solution for 1 hour and then shifted to destaining solution ( $40 \%$ methanol and $30 \%$ acetic acid) for 6-8 hours. Stained gels were photographed and approximate molecular weight of different peptide fractions were revealed by comparing with reference protein standards.

- Silk collection and silk solution preparation: Approximately $30 \mathrm{mg}$ of silk was extracted from the selected spider species, Crossopriza lyoni. To prepare stock silk solution, $30 \mathrm{mg}$ of silk was dissolved in $5 \% \mathrm{NaOH}$ by heating 
- Susceptibility test: For the examination of antibacterial potency of spider venom extracted from Pardosa oakleyi and spider silk recovered from Crossopriza lyoni, a susceptibility test following Kirby-Bauer Disk Diffusion method was used. Obvious inhibition zones were appeared against two Gram negative (i.e Acinetobacter sp. and Pasteurella sp.) and two are Gram positive (i.e Staphylococcus sp. and Streptococcus sp.) strains. These strains were cultured on nutrient Agar and maintained in liquid nutrient broth at $37^{\circ} \mathrm{C}$.

- Statistical analysis: The Mean and the Standard error of mean (Mean \pm SE) were computed using Minitab 14 . One way ANOVA followed by Tukey's test was applied to compare the zone of inhibitions of treatments using SPSS version 13 (Statistical Package for Social Sciences, 13).

\section{Results and Discussion}

Partial characterization of venom and determination of peptide fractions

During gel electrophoresis we compared resolved protein bands to protein bands of known molecular weight, alienated on same gel. When venom of $P$. oakleyi was analyzed through gel electrophoresis, five different protein bands of $155 \mathrm{kDa}, 140 \mathrm{kDa}$, $100 \mathrm{Da}, 65 \mathrm{kDa}$ and $43 \mathrm{kDa}$ were appeared. Band of $43 \mathrm{KDa}$ was most intense and broad among all bands followed by $100 \mathrm{kDa}, 70 \mathrm{kDa}$ and $155 \mathrm{KDa}$. Whereas band of $140 \mathrm{kDa}$ was less intense (Figure 1).

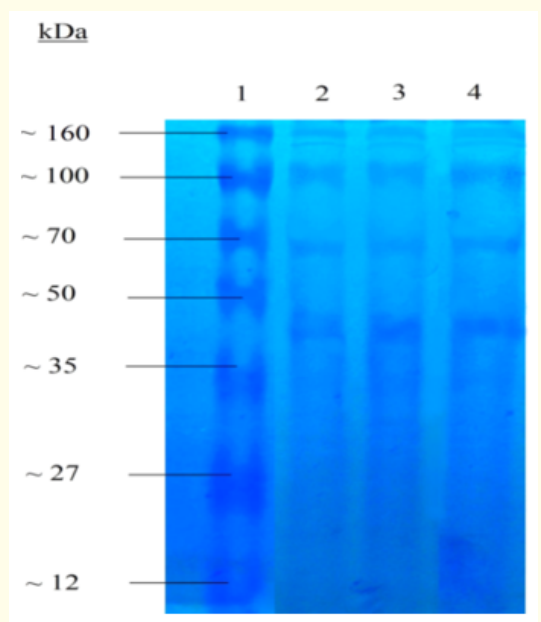

Figure 1: Electrophoretic separation of spider venom on SDSPolyacrylamide gel. Lane 1 was loaded with protein ladder. While Lane 2, 3, 4 contained venom of Pardosa oakleyi.

\section{Antibacterial activity}

The crude venom of Pardosa oakleyi did not exhibited bactericidal activity against any of the bacterial strain. Conversely by using silk solution, significant inhibition zones appeared in two of the tested bacterial strains i.e. Acinetobactor sp. and Streptococcus sp. The maximum zone of inhibition was obtained in case of Acinetobactor sp. (i.e., $3.523 \pm 0.433$ ) upon $100 \%$ silk treatment (Figure 2). The venom+silk solution induced reduced inhibition zones as compared to $100 \%$ silk. The inhibition zones shown by control groups were less significant as compared to both silk and venom+silk treated Acinetobactor sp. Similar results were obtained against Streptococcus sp. ( $\mathrm{P}<0.001)$ (Figure 3). However, in the case of Staphylococcus sp. and Pasteurella sp., both 100\% silk and venom+silk solution failed to produce significant inhibition zone.

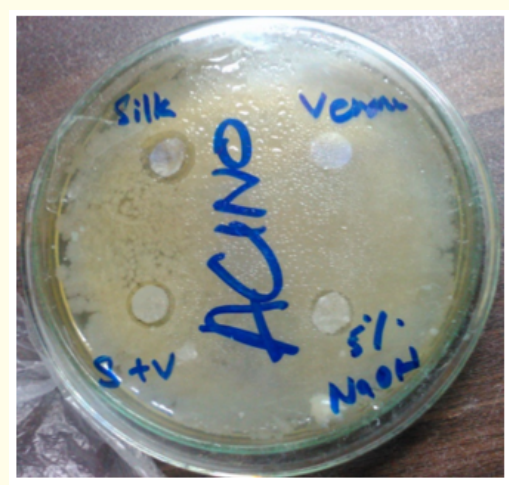

Figure 2: Zone of inhibition for Acinetobacter sp. produced by crude venom, $100 \%$ silk solution, venom+silk and $5 \% \mathrm{NaOH}$.

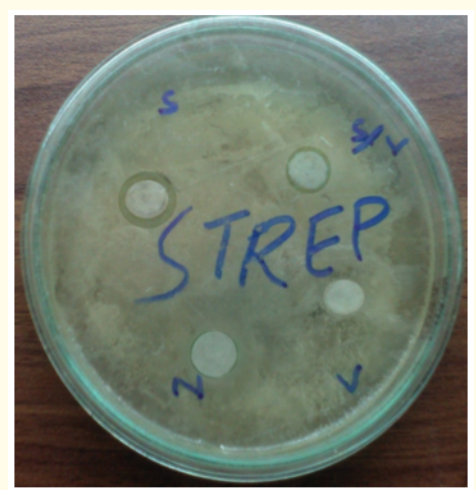

Figure 3: Zone of inhibition for Streptococcus sp. produced by crude venom, $100 \%$ silk solution, venom+silk solution and $5 \%$ $\mathrm{NaOH}$. 


\section{SDS-PAGE}

In present research, extracted venom from Pardosa oakleyi give rise to six bands (43-155kDa) on gel. Band of $43 \mathrm{kDa}$ was most significant and broad among all, which indicate high concentration of these peptides. Whereas as band of $140 \mathrm{kDa}$ was less significant. Effectiveness and mode of action of venom peptides of similar molecular weight as toxin is reported in available literature. It is described that the venom peptides of more than $10 \mathrm{kDa}$ molecular weight acts as toxin [26]. Moreover, cyto-insectotoxins of molecular weight 8kDa were extracted from Central Asian spider [27]. Similarly, venomous peptides of molecular weight $10 \mathrm{kDa}$ get identified in Selenocosmia huwena [28]. Venomous proteins of molecular weight ranging from 1 to $40 \mathrm{kDa}$ were reported in the venom of Loxosceles spiders [29]. Furthermore, Australian funnel-web spider, Hadronyche infensa contains noxious peptides having masses between 1,000 to 15,000 Da. While cytolytic peptides are generally smaller $(\sim 3 \mathrm{kDa})$ and the size of spider neurotoxins is fluctuated between $3-7 \mathrm{kDa}[30]$. That's why the peptide fraction present in the venom of selected species doesn't act like neurotoxin or cytotoxic.

\section{Effect of spider venom on cell culture}

Antibacterial potency of venom extracted from Agelena labyrinthica (Araneae: Agelenidae) is also reported [31]. It is demonstrated that Lycotoxins I and II (containing 25 and 27 amino acids respectively) isolated from the wolf spider Hogna carolinensis show potent lytic activity against Gram-negative bacteria (Escherichia coli) and yeast (Candida glabrata) [32]. Moreover, the peptides known as latarcins extracted from venom of Lachesana tarabaevi and spiderines of Oxyopes takobius venom exhibit cytolytic activity in gram-positive and gram-negative bacteria along with fungal cell i.e. yeast [33]. It is reported that the venom of $C$. salei comprised of lytic peptide having noticeable antibacterial potency against Gram positive bacteria i.e. Staphylococcus epidermidis and B. subtilis and Gram negative bacteria i.e. E. coli, Pseudomonas putida and Paracoccus denitrificans [34].

But still our current research reflects that crude venom of $\mathrm{Lyco}$ sids (Pardosa Hadronyche infensa) has no bactericidal effect on any of the tested strain. These contradictory results can be explained by presence of peptides of high molecular weight, these larger peptides cannot penetrate cell membrane. So, that's why they cannot exhibit cytolytic activities. Here one thing is noteworthy that the peptides present in venom of Pardosa oakleyi have higher molecular masses. These larger peptides cannot exhibit cytolytic activities as they cannot penetrate cell membranes.

\section{Effect of spider silk on bacterial culture}

Currently we examined spider silk for its antibacterial activity. Results reveal inhibitory effect of Crossopriza lyoni silk against Gram negative Acinetobactor sp. and Gram positive Streptococcus sp. Many researchers have discussed the possibility that spider silk can induce growth inhibitions in microbes [21,34,35]. Antimicrobial effect of spider silk against both Gram negative and Gram positive strains has been reported also [36]. According to a report, silk of Nephila pilipes possess antibacterial activity against gram negative E. coli and Pseudomonas aeruginosa as well as against gram positive Staphylococcus aureus [37]. These findings are in accordance with our research, indicating that the spider silk is potent for both Gram negative as well as Gram positive strains.

However, some studies have revealved that the spider silk only inhibits Gram positive strains. A study has shown that the Tegenaria domestica silk has inhibited the growth of the Gram positive bacterium [38]. Bacillus subtilis while has no effect on E. coli, a gram negative bacteria. Furthermore, the Lasiodora parahybana silk is shown to possess some antimicrobial activity against Gram positives [39]. This contradiction could be explained by the fact that the antimicrobial effect of silk of different spider species varies with the attributes, quality, type and the arrangement of amino acids in the silk fibre [38].

Our results also revealed that the spider silk did not inhibit the growth of Staphylococcus sp. and Pasteurella sp. This may be explained by the fact that our experimental set up was not sensitive enough to detect significant inhibitions against this microbe or it could be possible that like many other antimicrobial agents, our treatment has narrow spectra of activity and it generates differential inhibitions in different types of bacteria. The difference in inhibitions of bacteria could also be explained by the species phylogenetic distance.

Present study exposed that the spider silk more efficiently inhibit the growth of aerobic Gram negative Acinetobacter sp. The largest zones of inhibitions were noticed in Acinetobactor sp. pursued by 
Gram positive Streptococcus sp. Studies have revealed less adherence of surface of spider silk to Gram negative bacteria (P. aeruginosa and E. coli) in contrast to Gram positive bacteria (B. subtilis) $[40,41]$. Web silk of Nephila pilipes reported as greater inhibitory substance against Gram negative strains (Pseudomonas aeruginosa and Escherichia coli) as compared to Gram positive strains (Staphylococcus aureus) [37].

The findings of current research suggesting that the spider silk is more potent against Gram negative strain is contradictory to the findings of Mirghani., et al. (2012), Roozbahani., et al. (2014) and Wright and Goodacre (2012) whoshowed that Gram positive strains are more susceptible to the inhibitions induced by spider silk. This contradiction can be again explained by the differential antimicrobial activity of spider silk recovered from different spider species and the phylogenetic distances of selected bacterial strains.

\section{Conflict of Interest}

The authors declare that they have no conflict of interest.

\section{Bibliography}

1. Golkar Zhabiz., et al. "Bacteriophage therapy: a potential solution for the antibiotic resistance crisis". The Journal of Infection in Developing Countries 8.02 (2014): 129-136.

2. Sengupta Saswati., et al. "The multifaceted roles of antibiotics and antibiotic resistance in nature". Frontiers in microbiology 4 (2013): 47.

3. Spellberg Brad and David N Gilbert. "The future of antibiotics and resistance: a tribute to a career of leadership by John Bartlett". Clinical infectious Diseases 59 (2014): S71-S75.

4. Wright Gerard D. "Something old, something new: revisiting natural products in antibiotic drug discovery". Canadian Journal of Microbiology 60.3 (2014): 147-154.

5. Michael Carolyn Anne., et al. "The antimicrobial resistance crisis: causes, consequences, and management". Frontiers in Public Health 2 (2014): 145.

6. King Glenn F and Margaret C Hardy. "Spider-venom peptides: structure, pharmacology, and potential for control of insect pests". Annual Review of Entomology 58 (2013): 475-496.

7. Chen Yuxin., et al. "Rational design of $\alpha$-helical antimicrobial peptides with enhanced activities and specificity/therapeutic index". Journal of Biological Chemistry 280.13 (2005): 1231612329.
8. Cragg Gordon M and David J Newman. "Natural products: a continuing source of novel drug leads". Biochimica et Biophysica Acta (BBA)-General Subjects 1830.6 (2013): 3670-3695.

9. Windley Monique J., et al. "Spider-venom peptides as bioinsecticides". Toxins 4.3 (2012): 191-227.

10. Adams Michael E. "Agatoxins: ion channel specific toxins from the American funnel web spider, Agelenopsisaperta". Toxicon 43.5 (2004): 509-525.

11. Vassilevski AA., et al. "Molecular diversity of spider venom". Biochemistry (Moscow) 74.13 (2009): 1505-1534.

12. Saez Natalie J., et al. "Spider-venom peptides as therapeutics". Toxins 2.12 (2010): 2851-2871.

13. Escoubas Pierre., et al. "Venom landscapes: mining the complexity of spider venoms via a combined cDNA and mass spectrometric approach". Toxicon 47.6 (2006): 650-663.

14. Escoubas Pierre and Glenn F King. "Venomics as a drug discovery platform". Expert Review of Proteomics 6.3 (2009): 221224.

15. Escoubas Pierre., et al. "Isolation of a tarantula toxin specific for a class of proton-gated Na+ channels". Journal of Biological Chemistry 275.33 (2007): 25116-25121.

16. Gao T., et al. "PHLPP: a phosphatase that directly dephosphorylates Akt, promotes apoptosis, and suppresses tumor growth". Molecular Cell 18 (2005): 13-24.

17. Gellynck, Kris., et al. "Silkworm and spider silk scaffolds for chondrocyte support". Journal of Materials Science: Materials in Medicine 19.11 (2008): 3399-3409.

18. Bouzark k. "Web of intrigue”. Nature 519 (2005): 4-6.

19. Gomes S C., et al. "Functionalized silk biomaterials for bone regeneration". Semana de Engenharia. Guimarães 11 (2010): 1-2.

20. Roozbahani Hassan., et al. "Evaluation of antimicrobial activity of spider silk Pholcusphalangioides against two bacterial pathogens in food borne". International Journal of Advanced Biological and Biomedical Research 2.7 (2014): 2197-2199.

21. Heimer S. "Wunderbare Welt der Spinnen, Urania". Verlag Leipzig Jena Berlin (Urania) (1988). 
22. Cragg Stephanie J and Susan A Greenfield. "Differential autoreceptor control of somatodendritic and axon terminal dopamine release in substantia nigra, ventral tegmental area, and striatum". Journal of Neuroscience 17.15 (1997): 5738-5746.

23. Guerrero Belsy., et al. "Activities against hemostatic proteins and adrenal gland ultrastructural changes caused by the brown widow spider Latrodectusgeometricus (Araneae: Theridiidae) venom". Comparative Biochemistry and Physiology Part C: Toxicology and Pharmacology 151.1 (2010): 113121.

24. Frontali N., et al. "Purification from black widow spider venom of a protein factor causing the depletion of synaptic vesicles at neuromuscular junctions". The Journal of Cell Biology 68.3 (1976): 462-479.

25. Sambrook Joseph. "Commonly used techniques in molecular cloning”. Molecular cloning 3 (1987): E1-E39.

26. King Glenn F., et al. "A rational nomenclature for naming peptide toxins from spiders and other venomous animals". Toxicon 52.2 (2008): 264-276.

27. Vassilevski Panayot S. "Multilevel block factorization preconditioners: Matrix-based analysis and algorithms for solving finite element equations". Springer Science and Business Media (2008).

28. Liang Song-Ping., et al. "The presynaptic activity of huwentoxin-I, a neurotoxin from the venom of the Chinese bird spider Selenocosmiahuwena". Toxicon 38.9 (2000): 1237-1246.

29. Futrell Josephine M. "Loxoscelism". The American Journal of the Medical Sciences 304.4 (1992): 261-267.

30. Kuhn-Nentwig, Lucia, et al. "N-terminal aromatic residues closely impact the cytolytic activity of cupiennin 1a, a major spider venom peptide". Toxicon 75 (2013): 177-186.

31. Benli M and N Yigit. "Antibacterial activity of venom from funnel web spider Agelenalabyrinthica (Araneae: Agelenidae)". Journal of Venomous Animals and Toxins including Tropical Diseases 14.4 (2008): 641-650.

32. Yan Lizhen and Michael E Adams. "Lycotoxins, antimicrobial peptides from venom of the wolf spiderLycosacarolinensis". Journal of Biological Chemistry 273.4 (1998): 2059-2066.
33. Kozlov Sergey A., et al. "Latarcins, antimicrobial and cytolytic peptides from the venom of the spider Lachesanatarabaevi (Zodariidae) that exemplify biomolecular diversity". Journal of Biological Chemistry 281.30 (2006): 20983-20992.

34. Hisa Yasuo, et al. "Relationship of neuropeptides to nitrergic innervation of the canine laryngeal glands". Regulatory peptides 66.3 (1996): 197-201.

35. FAIRBROTHER Wayne J., et al. "The roles of ATP4- and Mg2+ in control steps of phosphoglycerate kinase". European journal of biochemistry 190.2 (1990): 407-414.

36. Chakraborty D and S Das. "Antibacterial activities of cobweb protein: R2127". Clinical Microbiology and Infection 15.4 (2009): S626-S627.

37. Amaley A., et al. "Antibacterial nature of $\mathrm{dm}$, ragline silk of Nephila pilipes (Fabricius, 1793)". Indian Journal of Arachnology 3 (2014): 8.

38. Wright Simon and Sara L Goodacre. "Evidence for antimicrobial activity associated with common house spider silk". BMC Research Notes 5.1 (2012): 326.

39. Wright Simon. The antimicrobial properties of spider silk. Diss. University of Nottingham, (2011).

40. Sharma Sumita. "A study on spiders as predators in the agro ecosystems". Munis Entomology and Zoology 9.1 (2014): 8083.

41. Mirghani Mohamed ES., et al. "Bt-201: Investigation of the spider web for antibacterial activity". 2nd Malaysian International Conference on Trends in Bioprocess Engineering (MICOTriBE 2012) 3 (2012).

\section{Volume 2 Issue 10 October 2019 (C) All rights are reserved by Iram Liaqat., et al.}

